\title{
On Fair and Efficient Power Control in CDMA Wireless Data Networks
}

\author{
George Alyfantis, Stathes Hadjiefthymiades, and Lazaros Merakos \\ Communication Networks Laboratory \\ Department of Informatics and Telecommunications, University of Athens \\ Athens 15784, Greece \\ \{alyf, shadj, merakos\}@di.uoa.gr
}

\begin{abstract}
We consider the uplink power control problem in a single cell, multi-user, CDMA wireless data system and formulate it as a cooperative game. We use the Nash bargaining solution concept, in order to determine the socially optimum solution, which is both Pareto efficient and fair. In our formulation, the BS plays the role of the arbitrator, i.e., solves the power control problem, and broadcasts the relevant information to all users in order to enforce convergence to the optimal operating point. The comparison of the cooperative scheme to the non-cooperative scheme shows significant reduction in the transmission power of the mobile terminals.
\end{abstract}

Keywords-CDMA, power control, game theory, Nash bargainng solution

\section{INTRODUCTION}

WCDMA has been widely adopted as the air interface technology for third generation (3G) networks [1]. WCDMA is based on Direct Sequence CDMA (DS-CDMA), which is a spread spectrum technology where user signals are spread over the entire transmission spectrum, and unique digital codes are used to separate them. It is well known that minimizing interference in CDMA, using power control, increases capacity, and also extends the battery lifetime of the mobile terminals [2], [3].

A popular approach to the power control problem is based on game theoretic models [6], [9]. In such models, users adjust their transmission power in a distributed manner, according to their selfish interests. However, solutions that are achieved, i.e., Nash equilibria (NE) [7], are typically not efficient from a social perspective [9]. The introduction of pricing may yield more efficient operating points, however, fairness is often compromised [9]. Hence, the determination of both efficient and fair operating points in the power control problem remains an open issue.

In this paper, we introduce a centralized power control scheme for CDMA networks, based upon cooperative game theory. Our intention is to, simultaneously, achieve fair and efficient operating points. Using the Nash bargaining solution (NBS) [8], we achieve a Pareto efficient and fair solution. The analysis that we carry out also proves that the solution provided in [12] is socially optimal, an issue that has been previously overlooked. In our formulation, the base station (BS) plays the role of the arbitrator, i.e., solves the power control problem, and broadcasts the relevant information to all users in order to enforce the optimal operating point. 
The rest of the paper is organized as follows. In Section II, we provide the formulation of the power control game, and introduce the concept of the NBS. In Section III, we provide the solution to the optimization problem that corresponds to the NBS of the power control game. An iterative algorithm for the arithmetic calculation of the NBS is studied in Section IV. Section V provides results regarding the performance of the proposed scheme. In Section VI, we discuss related prior work. Lastly, in Section VII, we provide our conclusions.

\section{PROBlEM AND GAME FormULATION}

Let $I=\{1, \ldots, N\}$ be the set of users who share the uplink bandwidth of a CDMA cell. User $i$ controls his transmitted power $p_{i}$, which is chosen from $S_{i}=[0,+\infty)$. Let $\boldsymbol{p}=\left(p_{1}, \ldots, p_{N}\right)$ be a typical strategy (i.e., transmitted power) profile vector in the strategy space $S=S_{1} \times \ldots \times S_{N}$. We assume that user preferences are expressed through a utility function, which quantifies the level of user satisfaction for using the wireless resources (e.g., achieved QoS vs. energy consumption).

Here, we adopt the popular utility function introduced in [9], which expresses the number of bits that are successfully received per unit of consumed energy. Let $L$ be the length (bits) of a user frame, $M$ the length of the frame with headers $(M>L)$, and $R$ the transmission rate $(\mathrm{b} / \mathrm{s})$. Then, the utility function $u_{i}: S \rightarrow \mathfrak{R}$ is

$$
u_{i}(\boldsymbol{p})=\frac{L R}{M p_{i}}\left(1-e^{-0.5 \gamma_{i}}\right)^{M} \quad(\text { bits/Joule }) .
$$

$\gamma_{i}$ that denotes the signal-to-interference ratio (SIR) as seen by user $i$, is calculated as follows:

$$
\gamma_{i}=\frac{W}{R} \frac{h_{i} p_{i}}{\sum_{k \neq i} h_{k} p_{k}+\sigma^{2}}
$$

$W$ is the chip rate, $W / R$ denotes the CDMA processing gain, $h_{\mathrm{i}}$ is the path loss from the mobile to the $\mathrm{BS}$, and $\sigma^{2}$ the additive white Gaussian noise (AWGN) power at the receiver.

\section{A. Non-cooperative Game Formulation}

In this section, we determine the NE of the game as in [9]. We first provide a definition of the NE.

Definition 2.1: A power vector $\boldsymbol{p}=\left(p_{1}, \ldots, p_{N}\right)$ is a NE of the power control game if, for every $i \in I$,

$$
u_{i}\left(p_{i}, \boldsymbol{p}_{-i}\right) \geq u_{i}\left(p^{\prime}{ }_{i}, \boldsymbol{p}_{-i}\right)
$$

for all $p_{i}^{\prime} \in S_{i}$, where $\boldsymbol{p}_{-i}$ is defined as the power vector that does not contain $p_{i}$

Thus, at a NE, given the power levels of the other users, no user can improve his utility level by making individual changes to his transmitted power. $\boldsymbol{p}^{*}=\left(p_{1}{ }^{*}, \ldots,{p_{N}}^{*}\right) \in S$ is said to constitute a NE [7] iff

$$
p_{i}^{*} \in \arg \max _{g \in S_{i}} u_{i}\left(g, \boldsymbol{p}_{-i}^{*}\right), \forall i \in I .
$$

The utility $u_{i}(\cdot)$ of user $i$ is maximized when its first derivative with respect to $p_{i}$ is zero, or,

$$
\frac{\partial u_{i}}{\partial p_{i}}=0.5 M \gamma_{i} e^{-0.5 \gamma_{i}}-\left(1-e^{-0.5 \gamma_{i}}\right)=0
$$


For $p_{i}=0$, we observe that $\partial u_{i} / \partial p_{i}=0$. However, it is easy to see that $p_{i}=0$ cannot be a maximizer of $u_{i}(\cdot)$. This leads to the fact that, at the NE, all users enjoy equal non-zero $\operatorname{SIR} \gamma^{*}$, which derives as a solution to the equation

$$
0.5 M \gamma+1=e^{0.5 \gamma}
$$

which can be readily solved numerically. Note that in order to have a feasible power vector, $\gamma^{*}$ must satisfy the following inequality [9]:

$$
N<1+\frac{W}{R \gamma^{*}}
$$

The fact that all users enjoy the same $\operatorname{SIR} \gamma^{*}$, leads to the result that all users reach the BS with the same power, i.e., $h_{i} p_{i}{ }^{*}=h_{j} p_{j}{ }^{*}$ $=q^{*}$ for every $i, j \in I$. From the above, the equilibrium utility $u_{i}^{*}$ for user $i$ is

$$
u_{i}^{*}=\frac{L R}{M p_{i}^{*}}\left(1-e^{-0.5 \gamma^{*}}\right)^{M}=h_{i} v\left(q^{*}\right),(6)
$$

where $v(q)=\left(1 / h_{i}\right) u_{i}\left(q / h_{1}, \ldots, q / h_{N}\right), \forall i \in I$.

Hence, at the equilibrium, all users achieve SIR $\gamma^{*}$, but transmit with different power depending on their path gain to the BS. As a result, a user that suffers great path losses consumes more energy in order to reach the equilibrium SIR. For this reason, user $i$ achieves utility $v\left(q^{*}\right)$ discounted by the corresponding path loss $h_{i}$.

Note that the discussed NE is fair, as all users achieve the same SIR (throughput). However, as reported in [9], this equilibrium is not Pareto optimal, i.e., it is possible to find another point, which yields strictly superior utility for all users. Specifically, it was shown that if all users simultaneously decrease their transmission power by a given factor, then all users may benefit by an increase in their utilities. A formal definition of Pareto optimality follows:

Definition 2.2: The point $\boldsymbol{u} \in U$, where $U$ is the set of achievable utilities, is said to be Pareto optimal if for each $\boldsymbol{u}^{\prime} \in U, \boldsymbol{u}^{\prime} \geq \boldsymbol{u}$, then $\boldsymbol{u}^{\prime}=\boldsymbol{u}$.

\section{B. Cooperative Power Control}

We use cooperative game theory in order to provide a fair and efficient solution to the power control game. User $i$, apart from his utility function $u_{i}$ defined on $S$, has also a minimum desired utility $u_{i}^{0}$, termed as status quo utility. This utility denotes the minimum utility that he can achieve without cooperation - here $u_{i}^{0}=h_{i} v\left(q^{*}\right)=u_{i}^{*}($ see $(6))$. We denote the status quo of the game by $\boldsymbol{u}^{0}=\left(u_{1}^{0}, \ldots, u_{K}{ }^{0}\right)$.

Since, in the considered game, the status quo is not Pareto optimal, a Pareto optimal solution would be acceptable by all users (all users would enjoy greater utility). One typical solution concept for obtaining Pareto optimal outcomes is the bargaining solution introduced by Nash [8], defined below.

Definition 2.3: A mapping $F: G \rightarrow \mathfrak{R}^{N}$, where $G$ denotes the set of achievable utilities with respect to the initial agreement point $\boldsymbol{u}^{\boldsymbol{0}}$, is said to be a NBS, if the following hold:

1) $F\left(U, \boldsymbol{u}^{0}\right) \in U_{0}$, where $U_{0}$ is the set of achievable utilities that are superior to the status quo utility.

2) $F\left(U, \boldsymbol{u}^{0}\right)$ is Pareto optimal. 
3) $F$ satisfies the linearity axiom if $\boldsymbol{\varphi}: \mathfrak{R}^{N} \rightarrow \mathfrak{R}^{N}, \boldsymbol{\varphi}(\boldsymbol{u})=\boldsymbol{u}^{\prime}$ with $u_{j}^{\prime}=a_{j} u_{j}+b_{j}, a_{j}>0, j=1, \ldots, N$, then $F\left(\boldsymbol{\varphi}(\boldsymbol{u}), \boldsymbol{\varphi}\left(\boldsymbol{u}^{0}\right)\right)=\boldsymbol{\varphi}(F(\boldsymbol{u}$, $\left.u^{0}\right)$.

4) $F$ satisfies the irrelevant alternatives axiom if $V \subset U,\left(V, \boldsymbol{u}^{0}\right) \in G$ and $F\left(U, \boldsymbol{u}^{0}\right) \in \mathrm{V}$, then $F\left(U, \boldsymbol{u}^{0}\right)=F\left(V, \boldsymbol{u}^{0}\right)$.

5) $F$ satisfies the symmetry axiom if $U$ is symmetric with respect to a subset $J \subseteq\{1, \ldots, N\}$ of indices (i.e., $\boldsymbol{u} \in U$ and $i, j \in J$, then if $u_{i}^{0}=u_{j}^{0}$ then $F\left(U, \boldsymbol{u}^{0}\right)_{i}=F\left(U, \boldsymbol{u}^{0}\right)_{j}$ for $\left.i, j \in J\right)$.

Each vector $\boldsymbol{p}$ in the bargaining solution set satisfies $u_{j}(\boldsymbol{p})>u_{j}^{0}, \forall j \in I$ and solves the following optimization problem.

$$
\max _{\boldsymbol{p}} \prod_{j=1}^{N}\left(u_{j}(\boldsymbol{p})-u_{j}^{0}\right), \quad \boldsymbol{p} \in\left\{\boldsymbol{r} \in S: \boldsymbol{u}(\boldsymbol{r})>\boldsymbol{u}^{0}\right\}
$$

\section{FAIR AND EFFICIENT POWER CONTROL}

In this section, we provide a characterization of the NBS of the power control game. Consider a linear transformation $\varphi: \Re^{N} \rightarrow$ $\mathfrak{R}^{N}$, where $\boldsymbol{\varphi}(\boldsymbol{u})=\boldsymbol{v}, v_{i}=\left(1 / h_{i}\right) u_{i}, \forall i \in I$. It is easy to see that the transformed utility functions $v_{i}$ will be of the following form:

$$
v_{i}(\boldsymbol{q})=\frac{L R}{M q_{i}}\left(1-e^{-0.5 \gamma_{i}}\right)^{M}, q_{i}=h_{i} p_{i}(7)
$$

The next lemma states that the NBS is an equal-SIR operating point.

Lemma 3.1: The NBS of the power control game, with the utility functions in (7), is characterized by equal SIR for all users.

Proof: From the definition of the utility function in (7), it is easy to see that the utilities of the users are symmetric. Moreover, we observe that status quo utilities are equal for all users, i.e., $v_{i}\left(q^{*}, \ldots, q^{*}\right)=v_{i}^{0}=v_{j}^{0}=v^{*}, \forall i, j \in I$. It is, then, straightforward from the fifth axiom of the NBS (i.e., the symmetry axiom) that the NBS of the given game should yield an operating point $\boldsymbol{q}$ where $v_{i}(\boldsymbol{q})=$ $v_{j}(\boldsymbol{q}), \forall i, j \in I$, which implies that $q_{i}=q_{j}=q$. Then, from (2), it is easy to verify that all users will enjoy the same SIR.

From the above, we may redefine the optimization problem whose solution is the NBS, as follows:

$$
\begin{aligned}
& \quad \max _{\boldsymbol{q}} f(\boldsymbol{q}), f(\boldsymbol{q})=\prod_{j=1}^{N}\left(v_{j}(\boldsymbol{q})-v_{j}^{0}\right), \\
& \boldsymbol{q} \in\left\{\boldsymbol{r} \in S: \boldsymbol{v}(\boldsymbol{r})>\boldsymbol{v}^{0}, q_{i}=q_{j}, \forall i, j \in I\right\}
\end{aligned}
$$

It is easy to see that, when $q_{i}=q_{j}=q, v_{i}(\boldsymbol{q})=v_{j}(\boldsymbol{q})=v(q)$, where

$$
v(q)=\frac{L R}{M q}\left(1-e^{-0.5 \gamma}\right)^{M}, \text { and } \gamma=\frac{W}{R} \frac{q}{(N-1) q+\sigma^{2}} .
$$

Hence, the problem $(P)$ reduces to the following, much simpler, problem:

$$
\text { (P) } \max _{q} v(q), \quad q \in\left\{r \in S_{j}: v(r)>v^{*}\right\} .
$$

The following lemma states that the objective function $v(q)$ of problem $\left(P^{\prime}\right)$ has a unique positive maximizer.

Lemma 3.2: There is a unique positive power $\widetilde{q}$ that maximizes function $v(q)$.

Proof: At the point(s) where function $v(q)$ is maximized the first-order optimality condition must hold, i.e., $d v(q) / d q=0$, or 


$$
\begin{aligned}
& \frac{L R}{M} \frac{0.5 \frac{W}{R} \frac{\sigma^{2} q}{\left[(N-1) q+\sigma^{2}\right]^{2}} M e^{-0.5 \gamma}-\left(1-e^{-0.5 \gamma}\right)}{q^{2}} \times \\
& \left(1-e^{-0.5 \gamma}\right)^{M-1}=0
\end{aligned}
$$

We observe that for $q=0$, the first-order optimality condition is satisfied. However, $v(0)=0$, while $v^{*}>0$, which violates the first axiom of the NBS, i.e., that each user has to achieve a utility strictly superior to his status quo utility. Thus, we may drop factors of the above equation that are zero for $q=0$, and, thus, derive the simpler condition below:

$$
\frac{W M \sigma^{2}}{2 R} \frac{q}{\left[(N-1) q+\sigma^{2}\right]^{2}}=e^{0.5 \gamma}-1(8)
$$

Equivalently, we may require that $r(q)=0$, where function $r(q)$ is defined as follows:

$$
r(q)=\frac{W M \sigma^{2}}{2 R} \frac{q}{\left[(N-1) q+\sigma^{2}\right]^{2}}-\left(e^{0.5 \gamma}-1\right)(9)
$$

We will now prove that the function $r(q)$ has a unique root in the interval $(0,+\infty)$. It is easy to see that the left-hand side of equation (8) is increasing in the interval $\left[0, \sigma^{2} /(\mathrm{N}-1)\right]$ and decreasing in the interval $\left[\sigma^{2} /(\mathrm{N}-1),+\infty\right)$. Moreover, the right-hand side of equation (8) is increasing in the interval $[0,+\infty)$.

The derivative of the function $r(q)$ is calculated as follows:

$$
\frac{d r(q)}{d q}=\frac{W}{2 R} \frac{\sigma^{2}}{\left[(N-1) q+\sigma^{2}\right]^{2}}\left[-M \frac{(N-1) q-\sigma^{2}}{(N-1) q+\sigma^{2}}-\sigma^{2} e^{0.5 \gamma}\right]
$$

We have that

$$
\left.\frac{d r(q)}{d q}\right|_{q=0}=\frac{W}{2 R \sigma^{2}}\left[M-\sigma^{2}\right] .
$$

In real-life situations, the AWGN power $\sigma^{2}<<1$ (Watt), in which case, we observe that the above quantity is positive, i.e., $r(q)$ is increasing, at $q=0$. Moreover, $r(0)=0$, hence, there is a sufficiently small positive scalar $\varepsilon$ for which $r(\varepsilon)>0$, which means that at $\varepsilon$ the left-hand side of (8) dominates the right-hand side of the equation. On the other hand, we observe that, for $q>[(\mathrm{M}-1) /(\mathrm{N}-$ 1)] $\sigma^{2}, r(q)<0$. This can be easily derived if we apply the known inequality $e^{x}-1>x, \forall x>0$, in (9). Thus, for values of $q$ in the interval $\left.[(\mathrm{M}-1) /(\mathrm{N}-1)] \sigma^{2},+\infty\right)$, the right-hand side of (8) dominates the left-hand side of the equation. As a result, there can be but one point of intersection of the left- and right-hand side quantities in (8), for $q>0$, i.e., function $r(q)$ has a single positive root.

The root, $\widetilde{q}$, of $r(q)$ is a point where the first-order optimality condition for problem $\left(P^{\prime}\right)$ is satisfied. Thus, since $\widetilde{q}$ is the unique positive root of $r(q)$, as already shown, $\widetilde{q}$ must be a global maximum or a global minimum of the function $v(q)$, for $q \in(0$, $+\infty)$. However, we showed that there is a small scalar $\varepsilon>0$ such that $r(\varepsilon)>0$, i.e., $v(q)$ is increasing, while for $q>[(M-1) /(N-1)] \sigma^{2}$, $r(q)<0$, i.e., $v(q)$ is decreasing. Hence, since $\widetilde{q} \in\left[\varepsilon,[(M-1) /(N-1)] \sigma^{2}\right]$, it is implied that point $\widetilde{q}$ is a global maximum of function $v(q)$ in the interval $(0,+\infty)$. 
In the following lemma, it is stated that the power $\widetilde{q}$ that maximizes the function $v(q)$ is a feasible solution of problem $\left(P^{\prime}\right)$, i.e., at $\tilde{q}$ every user achieves a utility that is strictly superior to his status quo utility.

Lemma 3.3: The positive scalar $\tilde{q}$ that maximizes function $v(q)$ is a feasible solution of problem $\left(P^{\prime}\right)$.

Proof: As already discussed in Section II, the non-cooperative power control game has a unique NE, at which all users achieve the same SIR $\gamma^{*}$, which solves (4). As a result, $q_{i}{ }^{*}=q_{j}{ }^{*}=q^{*}$, and $v_{i}\left(\boldsymbol{q}^{*}\right)=v_{j}\left(\boldsymbol{q}^{*}\right)=v\left(q^{*}\right), \forall i, j \in I$. Hence, it suffices to show that $v\left(q^{*}\right)<v(\widetilde{q})$. Since $\widetilde{q}$ is the maximizer of $v(q)$, it is obvious why inequality $v\left(q^{*}\right) \leq v(\widetilde{q})$ always holds. However, in order to complete the proof we need also to show that $v\left(q^{*}\right) \neq v(\widetilde{q})$ or, equivalently, that $q^{*} \neq \tilde{q}$, i.e., that $q^{*}$ is not a maximizer of the function $v(q)$. If we assume that $q^{*}$ maximizes $v(q)$, then $q^{*}$ must be a root of function $r(q)$. If we take $r(q)$ at $q^{*}$ and use (4), we have

$$
r\left(q^{*}\right)=\frac{M}{2} \gamma^{*}\left[\frac{-(N-1) q^{*}}{(N-1) q^{*}+\sigma^{2}}\right]
$$

Hence, since $q^{*}>0, r\left(q^{*}\right)<0$. This implies that $q^{*}$ can never nullify the derivative of $v(q)$, and, thus, can never be equal to $\widetilde{q}$. As a result, the status quo utility $v\left(q^{*}\right)$ is strictly inferior to the utility at point $\widetilde{q}$.

The next theorem states that the problem $\left(P^{\prime}\right)$ has a unique solution according to the NBS concept.

Theorem 3.1: There exists a unique equal-SIR NBS in the power control game, at which every user reaches the BS with power $\tilde{q}$ that is the positive root of (9).

Proof: The proof readily follows for Lemma 3.1, 3.2, and 3.3.

We, thus, proved that the NBS is an equal-SIR operating point, at which the user utility function is maximized. This result has been proposed in [12], however, it has not been examined whether such a solution is Pareto optimal, which is one of the results presented in this paper.

The BS executes this algorithm every time a new user attaches to the system, or when an active user disconnects. After such events, the updated optimal received power $\tilde{q}$ is broadcast so that every attached user $i$ adjusts his transmission power $\tilde{p}_{i}=\widetilde{q} / h_{i}$ accordingly ${ }^{1}$.

An important issue is how the BS can enforce such an operating point. The NBS provides Pareto optimality, but cannot guarantee stability; at the NBS, it is possible for a player to benefit in the detriment of the other users by making an appropriate change in his own strategy (contrary to the NE that is by definition a stable operating point).

A possible solution based on the "punishment" of deviating users has been proposed in [11]. The BS, upon detecting a user, which exceeds the optimum received power $\widetilde{q}$, increases intentionally his BER, by flipping a portion of the bits of the specific user. Hence, an increase in power only yields extra power consumption, with no BER improvement, which indicates that the utility of the user will decrease. This implies that no rational user would deviate from the NBS transmitted power.

\footnotetext{
${ }^{1}$ User $i$ is capable of inferring the path loss $h_{i}$ to the BS by means of beacon signals that are broadcast by the BS with a given power.
} 


\section{NBS DETERMINATION ALGORITHM}

In this section, based on the derived results, we provide a simple iterative algorithm for the determination of the discussed NBS. As already mentioned, the NBS of the power control game, i.e., the solution to the problem $\left(P^{\prime}\right)$, corresponds to the positive root of (9). In the proof of Lemma 3.2, it was shown that the positive root $\widetilde{q}$ of function $r(q)$, i.e., the NBS power, lies in the interval [ $\varepsilon$, $\left.[(M-1) /(N-1)] \sigma^{2}\right]$, where $\varepsilon$ is a sufficiently small positive scalar. Hence, since we have determined an interval where the root is located, we proceed with the definition of a simple numerical method for deriving the root. In the current study, we adopt the bisection [13] method for the determination of the pursued solution. The specific method guarantees convergence to the root, given that the function in question is continuous in the interval that is examined. Such condition is readily satisfied by function $r(q)$.

Let $q_{a}$ and $q_{b}$ be positive scalars, such that $q_{a}<\tilde{q}<q_{b}$. Below we summarize the bisection-based NBS determination algorithm:

1. Set $q_{a}=0$, and $q_{b}=[(M-1) /(N-1)] \sigma^{2}$,

2. Set $q_{c}=\left(q_{a}+q_{b}\right) / 2$,

3. If $r\left(q_{c}\right)=0$, then go to step 6 , else go to step 4 ,

4. If $r\left(q_{c}\right) r\left(q_{b}\right)<0$, then set $q_{a}=q_{c}$, else set $q_{b}=q_{c}$,

5. If $\left|q_{b}-q_{a}\right|<\delta$, go to step 6, else go to step 2,

6. $\tilde{q}=q_{c}$. Finish.

The constant $\delta$ in step 6 is a sufficiently small positive scalar, used as a termination criterion of the iterative algorithm. Note that the choice of the initial value of $q_{a}$ in step 1 is not in accordance with the discussion above regarding the interval where the root is located, i.e., that $q \neq 0$. However, it can be easily observed that in the algorithm given above, $q_{c}$ may approach arbitrarily close to 0 , but never reaches it, i.e., $q_{c}$ always stays in the interval $\left(0,[(M-1) /(N-1)] \sigma^{2}\right]$.

The main advantage of the bisection method is its simplicity, and that convergence is guaranteed, given an interval that includes the root, and that the examined function is continuous in this interval. However, convergence is not very fast. There are other more elaborate methods for finding the roots of a function, which, in general, converge much faster. For the considered problem, we have assessed the performance of two other methods, namely the linear interpolation (regula falsi) and the Newton-Raphson method [13]. The linear interpolation always converges to the root but not always as fast as the bisection method, the speed of which is not affected by the number of users $N$. The Newton-Raphson converges much faster than the bisection and the linear interpolation method, however, convergence is not always achieved as the required conditions are not always met for the considered problem. Especially for small values of $N$ the Newton-Raphson method systematically fails to converge.

\section{PERFORMANCE ANALYSIS}

In this section, we provide numerical results from the performance analysis of the proposed scheme. Table I gives the parameters used in our analysis, which are similar to [9]. 
TABLE I. THE LIST OF PARAMETERS FOR THE SIMULATED SINGLE-CELL CDMA SYSTEM

\begin{tabular}{|c|c|}
\hline $\boldsymbol{p}_{\boldsymbol{m a x}}$, maximum power constraint & 2 Watts \\
\hline $\boldsymbol{M}$, total number of bits per frame & 80 \\
\hline $\boldsymbol{L}$, number of information bits per frame & 64 \\
\hline $\boldsymbol{W}$, spread spectrum bandwidth & $10^{6} \mathrm{~Hz}$ \\
\hline $\boldsymbol{R}$, bit rate & $10^{4} \mathrm{bits} / \mathrm{second}$ \\
\hline $\boldsymbol{\sigma}^{2}$, AWGN power at the receiver & $5 \times 10^{-15}$ Watts \\
\hline modulation technique & non coherent FSK \\
\hline
\end{tabular}

The discussed power control game with the given parameters, at the NE, achieves a SIR value $\gamma^{*} \approx 12.42$, and from (5) it can support up to 9 users [9]. Hence, we compare the two different approaches (non-cooperative and cooperative schemes) for user population $N \in\{2, \ldots, 9\}$.

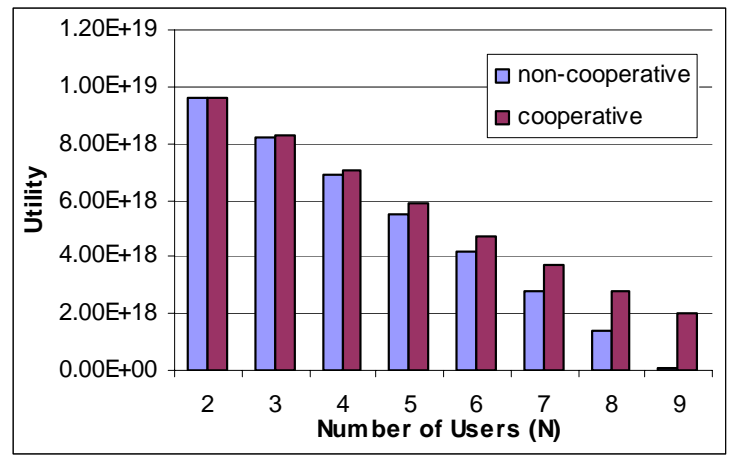

Figure 1. Utility achieved at the NE and NBS operating points

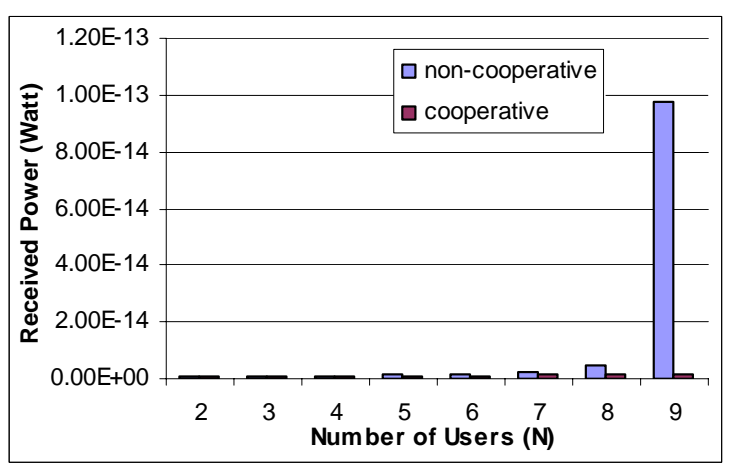

Figure 2. Received power at the NE and NBS operating points 


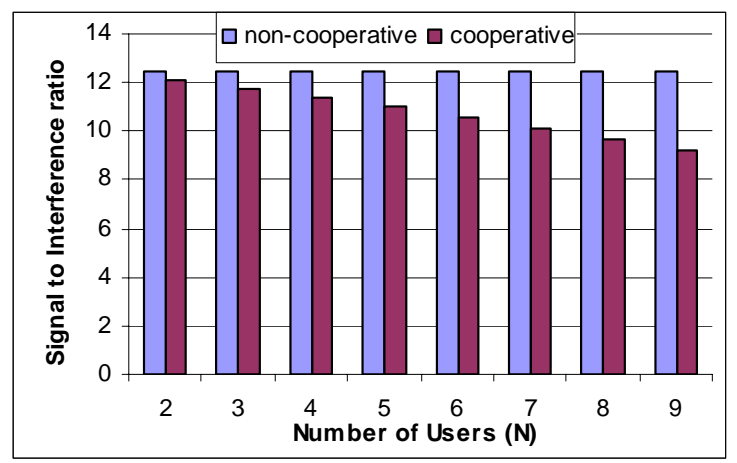

Figure 3. Signal-to-interference ratio (SIR) at the NE and NBS operating points

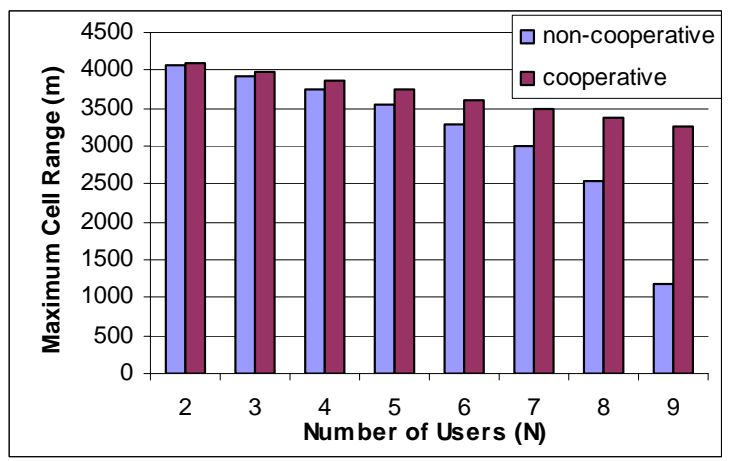

Figure 4. Maximum cell radius at the NE and NBS operating points

As shown in Fig. 1, the user utility (regardless of path losses, i.e., $v(q)=\left(1 / h_{i}\right) u_{i}(q, \ldots, q)$, for each $\left.i \in I\right)$ achieved with the proposed cooperative solution is significantly greater compared to the non-cooperative solution. This is due to the fact that users have to reach the BS with significantly lower power, as depicted in Fig. 2. Notice that this power reduction becomes apparent as the number of users increases, which is due to the inefficiency of the non-cooperative power control scheme, when the user population approaches its limit (see (5)). However, as users transmit with lower power with the cooperative scheme, the achieved SIR is also reduced, i.e., users enjoy lower throughput, compared to the non-cooperative scheme, as shown in Fig. 3. Nevertheless, such deterioration is rather minor compared to the major energy savings that are achieved.

We also study the system performance regarding the maximum cell radius. We assume a simple path loss model, as in [10], i.e., $h_{i}=K_{1} / d_{i}^{4}$, where $K_{1}=0.097$ and $d_{i}$ denotes the distance between the user and the BS. Given that every user can transmit with a power bounded by a maximum value $p_{\max }$ (see Table I), the distance $d_{i}$ from which user $i$ can reach the BS is also bounded. Let $d_{\max }$ denote this maximum distance, which is easily calculated as

$$
d_{\max }=\sqrt[4]{\frac{K_{1} p_{\max }}{q}}
$$

where $q$ is $q^{*}$ or $\tilde{q}$ depending on whether we examine the system at the NE or the NBS operating point. Fig. 4 shows that the cooperative scheme achieves a considerable increase in the maximum cell range compared to the non-cooperative scheme. 


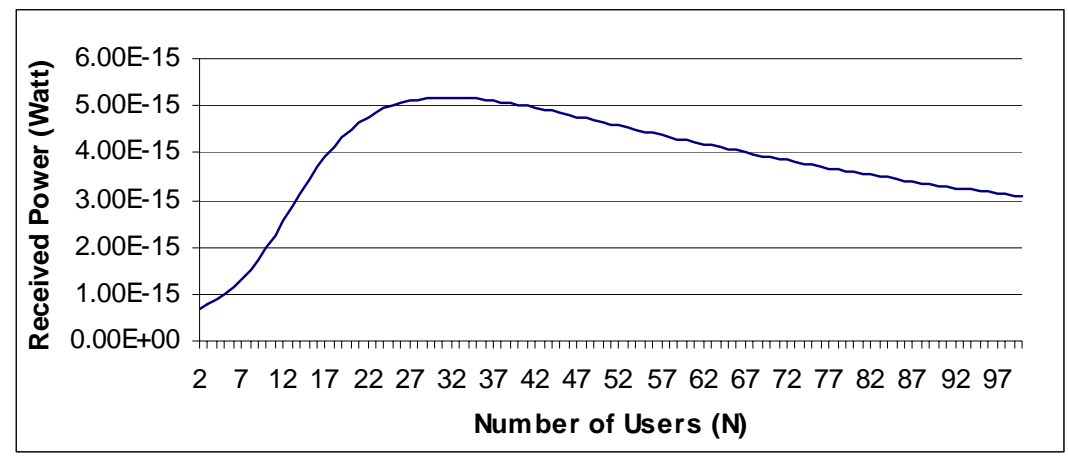

Figure 5. NBS Received power

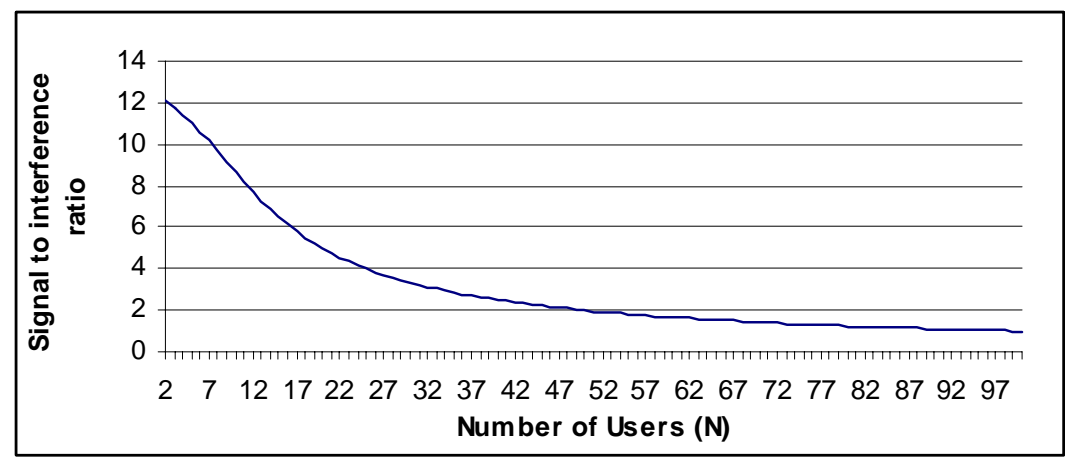

Figure 6. NBS Signal to interference ratio

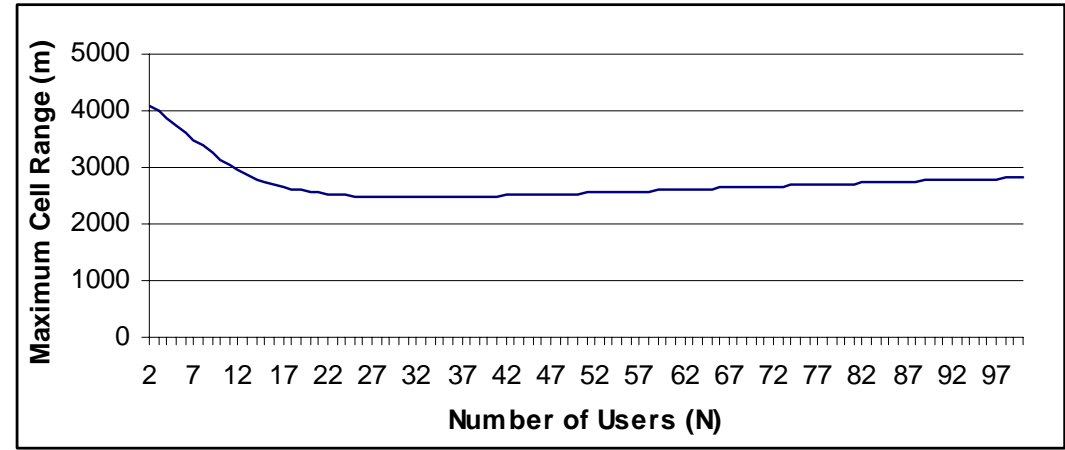

Figure 7. NBS Maximum cell radius

Another important feature of the cooperative scheme is that there is no limit in the number of users in the cell, as is the case of the non-cooperative scheme (see equation (5)). In Fig. 5, we see the NBS received power for a population of users ranging from 2 to 100. Fig. 6 depicts the achieved SIR. We observe that, as the number of users increases, the SIR approaches 0 . This is anticipated, because the capacity of a CDMA system is finite, thus, as more users share the available bandwidth, the individual share that they receive becomes smaller. Lastly, Fig. 7 depicts the maximum cell range achieved with the cooperative solution. 


\section{RELATED WORK}

In this section, we provide a brief overview of related work on game theoretic power control in CDMA networks. In [9], the power control problem has been formulated as a non-cooperative game, with a unique, however, inefficient NE. With the introduction of pricing, a Pareto improvement is achieved, but a Pareto optimal operating point, still, was not reached. Moreover, the fairness characteristic of the NE (equal SIR for all users) is compromised. Mobile stations that encounter high path loss are charged more and receive fewer resources, compared to users close to the BS. This is termed "near-far unfairness" [4]. Similar approaches are described in [4], [5], and [10].

In [11], the concept of refereed power control is introduced. The BS computes an optimum power allocation that then communicates to the users, which transmit accordingly. Cheating users (i.e., users that transmit with higher power) are punished - a portion of the user data bits is flipped. In [12], the notion of network assisted power control is introduced. Conceptually, it is the same approach as the one proposed in [11]. In both [11] and [12], the optimum power allocation is defined as an equal SIR allocation. The optimum operating point is the one that maximizes the user utility function (for equal received powers, or SIRs). However, the works in [11] and [12] lack a formal analysis on the efficiency of the proposed results. Specifically, the authors in [11] conjecture that the resulting power allocation is Pareto optimal. In [12], such issue is not addressed. Our main contribution is the methodology followed for deriving the optimal power allocation, as we employed the NBS that by definition yields Pareto optimal and fair operating points. Moreover, in contrast to previous relevant works, we studied the implementation details of the considered scheme and provided a low complexity algorithm for the NBS operating point determination.

\section{CONCLUSIONS}

In this paper, we have proposed a cooperative solution to the power control problem for CDMA wireless data networks, based on the Nash bargaining solution. Such a solution, contrary to non-cooperative game settings, guarantees Pareto optimal and fair outcomes. Specifically, we have achieved a solution where all users enjoy the same SIR.

The anticipated Pareto improvement achieved through the cooperative scheme was evident, especially when the noncooperative scheme reached its user population limits. We have also noticed that the operating points of the proposed scheme are characterized by significantly reduced transmitted power, and a rather limited decrease of the SIR. This resulted to a drastic decrease in energy costs, while the achieved throughput was slightly compromised. Furthermore, due to the transmitted power decrease, the maximum range of the cell was increased compared to the non-cooperative case. We concluded the study of the cooperative solution by investigating its implementation details. In the future, we would like to study the tradeoff between the number of admitted users and the obtained system performance. Moreover, we plan to extend our study to multi-cell environments and apply findings from cooperative game theory in order to enhance existing power control schemes.

\section{ACKNOWLEDGEMENTS}

The first author would like to thank the Alexander S. Onassis Public Benefit Foundation for its financial support.

\section{REFERENCES}

[1] H. Holma and A. Toskala, "WCDMA for UMTS", Wiley, New York, 2002.

[2] R. D. Yates, “A framework for uplink power control in cellular radio systems”, IEEE JSAC, 13(7), pp. 1341-1347, 1995. 
[3] J. Zander, "Performance of optimum transmitter power control in cellular radio systems", IEEE Transactions on Vehicular Technology, 41(1), pp. 57-62, 1992.

[4] M. Xiao, N. B. Shroff and E. K. P. Chong, “A Utility-based Power-Control Scheme in Wireless Cellular Systems”, IEEE/ACM Transaction on Networking, vol. 11, No 2, pp. 210-221, 2003

[5] T. Alpcan, T. Başar, R. Srikant and E. Altman, "CDMA Uplink Power Control as a Noncooperative Game", Wireless Networks 8, pp. 659-670, 2002

[6] H. Ji and C. Huang, "Non-cooperative uplink power control in cellular radio systems", Wireless Networks, (4), pp. 233-240, 1998.

[7] D. Fudenberg and J. Tirole, "Game Theory”, MIT Press, Cambridge (MA), 1991.

[8] J. F. Nash, "The Bargaining Problem", Econometrica, 18, pp. 155-162, 1950

[9] C. U. Saraydar, N. B. Mandayam and D. J. Goodman, "Efficient power control via pricing in wireless data networks", IEEE Transactions on Communications, Vol. 50, pp. 291 - 303, 2002

[10] S. Gunturi and F. Paganini, "Game theoretic approach to power control in cellular CDMA", Proc. IEEE Vehicular Technology Conference, Orlando, Oct 2003.

[11] A. B. MacKenzie and S. Wiker. "Game Theory in Communications: Motivation, explanation and application to power control", Proc. IEEE Globecom, November 2001.

[12] D. J. Goodman and N. B. Mandayam, "Network Assisted Power Control for Wireless Data", Kluwer Mobile Networks and Applications 6, pp. 409-415, 2001

[13] S. C. Chapra and R. P. Canale, "Numerical Methods for Engineers", McGraw-Hill, 1989 